Ophthalmologe 2009 · 106:674-675

DOI 10.1007/s00347-009-1970-9

Online publiziert: 18. Juli 2009

(c) Springer Medizin Verlag 2009

\author{
E.M. Hoffmann \\ Universitäts-Augenklinik Mainz
}

\title{
Neue Möglichkeiten in der Glaukomdiagnostik
}

heute noch zum wichtigsten Risikofaktor der Glaukomerkrankung. Jedoch weisen ca. 30-50\% aller Glaukompatienten einen Augeninnendruck innerhalb des statistischen Normbereichs auf. Solche Patienten werden daher durch Tonometrie alleine nicht erkannt. Da das Glaukom eine kontinuierlich voranschreitende Erkrankung ist und der Augeninnendruck als alleinige diagnostische Maßnahme also nicht ausreicht, sind weitere diagnostische Verfahren zur Früherkennung und Verlaufskontrolle der Erkrankung notwendig:

Die fundoskopische Papillenbeurteilung an der Spaltlampe erfordert Erfahrung und ist subjektiv. Aufgrund der hohen physiologischen Variabilität der Papillenmorphologie ist die Diagnose einer glaukomatösen Papillenschädigung oft schwierig. Außerdem sind die Strukturen der Papille wie etwa die Exkavation oder der neuroretinale Randsaum von der absoluten Papillengröße abhängig. Bei einer großen Papille $\left(>3 \mathrm{~mm}^{2}\right)$ kann die Exkavation entsprechend groß sein, ohne dass eine pathologische Ausdünnung des neuroretinalen Randsaums und damit eine glaukomatöse Schädigung vorliegt. Umgekehrt kann bei einer kleinen Papille $\left(<2 \mathrm{~mm}^{2}\right)$ schon bei kleiner Exkavation ein ausgeprägter Glaukomschaden bestehen, der dann übersehen werden kann. Die Papille und die retinalen Nervenfasern können auch anhand von Stereofotos oder RNFL-Fotos beurteilt werden. Diese Methoden sind zwar z. T. aufwändig, zeigen aber eine hohe Reproduzierbarkeit und Zuverlässigkeit in der Diagnose und Verlaufskontrolle. Laserbasierte Diagnoseverfahren wie die konfokale Scanning-Laser-Ophthalmoskopie, die Scanning-Laser-Polarimetrie und die optische Kohärenztomographie ermöglichen die dreidimensionale Darstellung der Papille und der retinalen Nervenfaserschicht. Die Reproduzierbarkeit der Verfahren ist hoch, es ist oftmals keine Pupillenerweiterung notwendig, die Analyse geschieht automatisiert und schnell. Die Messung ist quantitativ und erlaubt eine Verlaufskontrolle. Aber es gibt auch einige Nachteile: Die automatisch erstellten Resultate sind nur von einem erfahrenen Untersucher interpretierbar, und es wird viel Information gegeben, die für den Anwender häufig verwirrend ist. Weiterhin sind diese Instrumente teuer in der Anschaffung und bei schnell wechselnden Technologien ein „Fass ohne Boden“.

Die Entwicklung der automatischen Perimetrie in den späten 7oer Jahren erweiterte das diagnostische Spektrum des Augenarztes und bot die Möglichkeit, einen standardisierten Test für die Diagnostik des Glaukoms in Praxis und Klinik anzuwenden. Durch die Automatisierung wurde außerdem eine Unabhängigkeit von einem geschulten Gesichtsfeldassistenten erlangt, die bei der manuellen Perimetrie in hohem Maße bestand. Durch Standardisierung der Testobjekte (Leuchtdichte und Leuchtstärke), der Hintergrundleuchtdichte und der Patientenfixation wurde die Perimetrie besser reproduzierbar. Computergestützte Perimetrie hat weitere Vorteile wie die randomisierte Präsentation verschiedener Lichtpunkte, die Abschätzung der Zuverlässigkeit eines Patienten und die statistische Auswertung der Daten. Dennoch ist die hohe Variabilität zwischen verschiedenen Gesichtsfelduntersuchungen ein Problem geblieben.

Ein Gesichtsfelddefekt beim Glaukom tritt oftmals zuerst entlang der peripheren Grenzen auf. Das zugrunde liegende morphologische Korrelat des Gesichtsfeldde- 
fekts ist ein Schaden der Ganglienzellen und ihrer Axone. Der Initialschaden betrifft typischerweise ein oder mehrere Axone der retinalen Ganglienzellen. Dieser Schaden spiegelt sich dann bei Voranschreiten der Erkrankung in einem lokalisierten Gesichtsfeldausfall wieder. Es gibt Hinweise darauf, dass Axone großen Durchmessers als erstes geschädigt werden. Hier spielt die Frequenzverdopplungsperimetrie eine Rolle. Einen typischen frühen Schaden kann man im Gesichtsfeld nicht leicht erkennen, da es vor Auftreten typischer Gesichtsfelddefekte oft erst zu einer Zunahme der Fluktuation der retinalen Sensitivität bei der Untersuchung kommt. Möglicherweise ist die Frequenzverdopplungsperimetrie geeignet, besonders frühe Schäden zu detektieren.

Das Verständnis der Glaukomerkrankung und seiner Diagnostik hat sich in den letzten Jahrzehnten dramatisch geändert. Das folgende Leitthema erläutert die aktuellen klinischen Diagnoseverfahren, die zur Diagnostik und Verlaufskontrolle der Glaukomerkrankung eingesetzt werden.

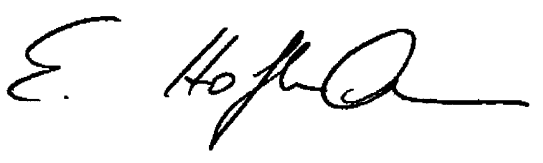

Esther Hoffmann

\section{Korrespondenzadresse}

\section{PD Dr. E.M. Hoffmann}

Universitäts-Augenklinik Mainz

Langenbeckstraße 1, 55131 Mainz

ehoffman@uni-mainz.de

Interessenkonflikt. Die korrespondierende Autorin weist auf folgende Beziehung/en hin: Referententätigkeit für Carl Zeiss Meditec und Heidelberg Engineering.

\section{Exklusiv für Abonnenten und Gesellschaftsmitglieder :}

\section{Nutzen Sie das Online-Archiv von Der Ophthalmologe}

Ihre Vorteile:

- Komfortable und schnelle Recherche nach Themen, Autoren, Suchbegriffen

— Ob unterwegs oder am eigenen PC: Zugriff überall und jederzeit

- Online First: Lesen Sie die aktuellsten Beiträge schon vor Erscheinen des gedruckten Heftes online

\section{Registrieren Sie sich jetzt unter}

www.DerOphthalmologe.de

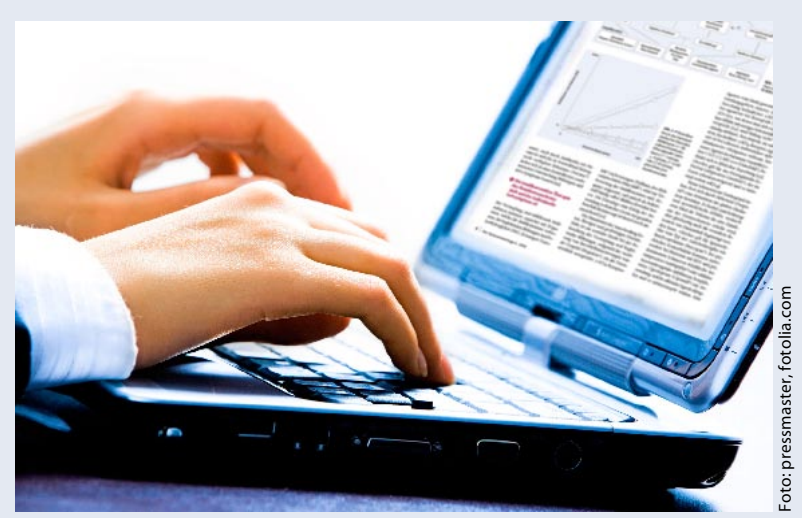

